einfaches Modell kann, wie in diesem Fall, einen unheimlich reichhaltigen Schatz an Ergebnissen liefern. Und das ist es, was mich letztlich an der Anwendung der Mathematik in den Umweltwissenschaften reizt.

Ist Mathematik in diesem Sinne für Sie ein Instrument oder mehr?

Mathematik ist für mich mehr! Sie ist eine Art und Weise, wie ich denke, wie ich an Sachen herangehe. Die zeigt sich insbesondere in der Phase, in der ich die mathematischen Modelle konzipiere. Und genau in dieser Vorgehensweise, in diesem Systemverständnis, liegt für mich die eigentliche Leistung der Mathematik.

Würden sie jungen Mathematikerinnen und Mathematikern, die sich für das Anwendungsgebiet Ökologie interessieren, über das Mathematikstudium hinaus noch ein Fachstudium empfehlen?

Im Prinzip reicht ein Mathematikstudium. Aber man sollte während des Studiums über den Tellerrand hinausschauen. Es reicht schon, ein echtes Interesse an einer praktischen Fragestellung zu haben. Und das muss nicht mal unbedingt eine ökologische Fragestellung sein. Eine unserer beiden Mathematikerinnen hier hat zum Beispiel für ihre Diplomarbeit Feldversuche eines Agrarforschungsinstituts analysiert und dabei neue Methoden der Statistik ausprobiert. Das war statistisch interessant, auBerdem zählte für sie, dass sie reale Datensätze verwenden konnte. Die andere hat im Rahmen eines Auslandsaufenthalts in Australien Planungs- und Optimierungsmodelle aus der Ökonomie genommen, um Naturschutzflächen zu planen und Kosten optimal auszuweisen. Ihre Arbeitsgruppe an der Uni hat den gesamten Naturschutzplan für das Great Barrier Reef vor der Nordküste Australiens umgesetzt. Man kann Mathematik in so unglaublich vielen Bereichen anwenden, nicht nur im Banken- und Versicherungssektor. Aber man braucht eine gewisse Offenheit, um dorthin zu gelangen.

Kristina Vaillant ist freie Journalistin in Berlin und arbeitet regelmäBig für das Medienbüro der Deutschen Mathematiker-Vereinigung. http://www.vaillant-texte.de

\title{
Ein neuer 4-regulärer Streichholzgraph
}

Mike Winkler

Als Streichholzgraphen bezeichnet man planare Graphen, deren Kanten Einheitslänge besitzen und die sich nicht überschneiden. Ihren Namen verdanken solche Graphen dem Umstand, dass man sie auch mit gleichlangen Streichhölzern auf einer flachen Oberfläche nachbilden kann. Regulär ist ein solcher Graph genau dann, wenn an jeden seiner Knoten gleich viele Kanten grenzen. Der kleinste bekannte 4-reguläre Streichholzgraph besitzt 104 Kanten und wurde 1986 von Heiko Harborth vorgestellt und auch nach ihm benannt. Ob es noch klei- nere solche Graphen gibt, ist nach wie vor ein offenes Problem.

Nachfolgend soll die Konstruktion eines neuen 4regulären Streichholzgraphen mit I I 4 Kanten vorgestellt werden. Dieser Graph ist - nach dem Harborth-Graphen mit 104 Kanten - das zweitkleinste Beispiel eines 4regulären Streichholzgraphen und unterbietet seinen Vorgänger, der diesen Rekord immerhin 30 Jahre hielt, um sechs Kanten. Wie sich der neue Graph in wenigen
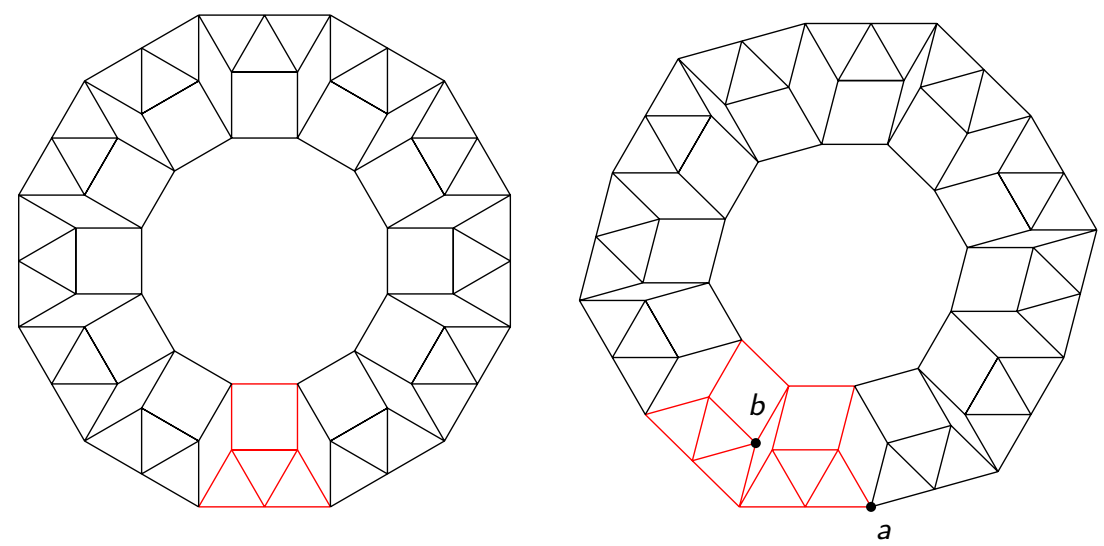

Abbildung I. 4-reguläre Streichholzgraphen mit I 20 Kanten 

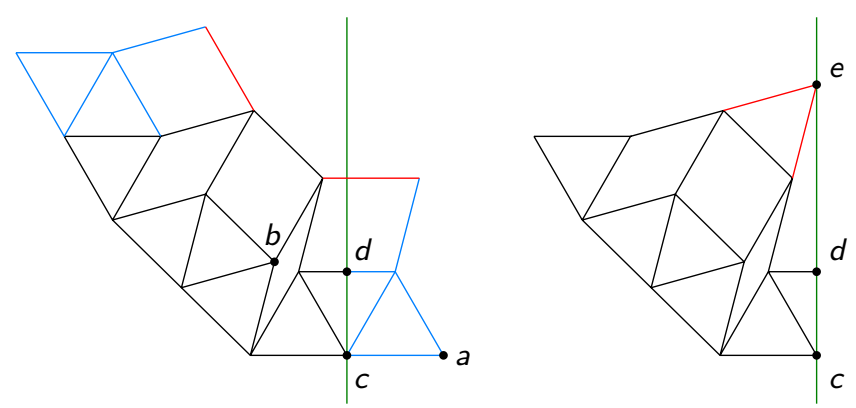

Abbildung 2. Die Konstruktion des Triplet-Kite

Schritten aus dem alten Graphen konstruieren lässt, soll hier gezeigt werden.

Der bisher zweitkleinste 4-reguläre Streichholzgraph besitzt 120 Kanten und besteht aus zwölf identischen Teilgraphen (Abb. I links). Dieser Graph besitzt die Symmetrie eines regelmäßigen I2-Ecks und ist flexibel. Er kann stetig verformt werden (wie in Abb. I rechts) und hat dann nur noch die Symmetrie eines regelmäßigen 6-Ecks.

Für das Folgende wählen wir nun die Verformung derart, dass die Strecke zwischen den Knoten $a$ und $b$ genau 2 Kantenlängen misst. Man kann sich den verformten Graphen vorstellen als Vereinigung von sechs Kopien des (in Abb. I rechts) rot markierten Teilgraphen, sodass je zwei zyklisch aufeinander folgende Kopien zwei Knoten gemeinsam haben. Dieser rote Teilgraph wird im Folgenden modifiziert wie in Abb. 2 gezeigt. Hierzu zieht man eine senkrechte Gerade durch den Knoten $c$ und den Kantenmittelpunkt $d$ (Abb. 2). Dann eliminiert man die neuneinhalb blauen Kanten und dreht die beiden roten Kanten an die senkrechte Gerade, auf der sie sich in Punkt e treffen. Im nächsten Schritt spiegelt man diese Figur an der senkrechten Gerade und erhält damit einen neuen Teilgraphen aus 22 Knoten und 4I Kanten, den wir Triplet-Kite genannt haben (Abb. 3).

Der Triplet-Kite ist ein starrer Graph und kann nicht verformt werden, ohne die Kantenlänge zu ändern. Dieser Graph besitzt eine ganz wunderbare geometrische Eigenschaft. Verbindet man nämlich die Kantenmittelpunkte $v$ und $w$ und konstruiert mit dieser Strecke ein gleichseitiges Dreieck, dann verlaufen die beiden Strecken $[v, z]$ und $[w, z]$ jeweils genau durch den Kantenmittelpunkt $x$ bzw. $y$. Daraus folgt, dass sich drei Triplet-Kites so kombinieren lassen, dass ihre äußeren Dreiecke jeweils paarweise genau übereinander liegen. Das führt uns schließlich zu unserem neuen minimalen 4-regulären Streichholzgraphen mit II4 Kanten.

Für weitere Details siehe http://tinyurl.com/zbee6aq.

\section{Literatur}

Sascha Kurz, Rom Pinchasi: Regular matchstick graphs. In: American Mathematical Monthly II8, Nr. 3, 20II, S. 264-267

Mike Winkler, Peter Dinkelacker, New minimal 4/n-regular matchstick graphs, 2016, arXiv:1604.07/34

Mike Winkler, Ernst-Abbe-Weg 4, 45657 Recklinghausen mike.winkler@gmx.de

Mike Winkler (Jg. 1974) ist selbstständiger Klavier- und Gitarrenlehrer sowie mathematischer Autodidakt mit Interessensschwerpunkt Collatz-Vermutung und Streichholzgraphen. Er plant, im Herbst 2016 ein Mathematikstudium aufzunehmen. Mathematik-Homepage: www.mikewinkler.co.nf

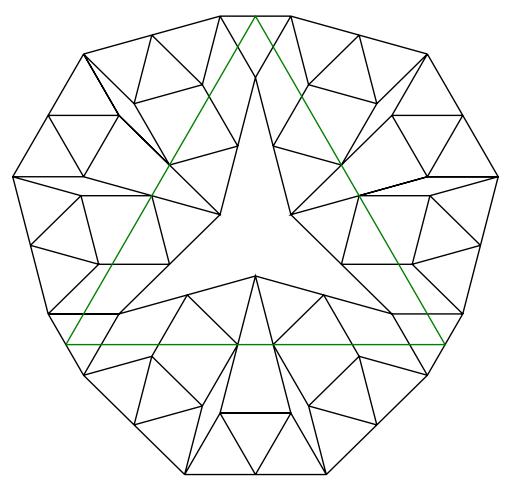

Abbildung 3. Drei Triplet-Kites bilden den neuen Graphen 